\title{
Synthesis of novel silica-polyimide nanocomposite films using aromatic-amino modified silica nanoparticles: Mechanical, thermal and morphological investigations
}

\author{
R. V. Ghorpade*, C. R. Rajan, N. N. Chavan, S. Ponrathnam \\ Polymer Science and Engineering Division, CSIR-National Chemical Laboratory, Dr. Homi Bhabha Road, Pune- 411008 \\ India
}

Received 13 October 2014; accepted in revised form 9 December 2014

\begin{abstract}
Silica nanoparticles were modified with aromatic amino groups and modified-silica/polyimide composite films were prepared using them. 3,3',4,4'-Benzophenone tetracarboxylic dianhydride (BTDA) and 4,4'-oxydianiline (ODA) were used as precursors for polyimide matrix. The structures of the modified nanoparticles and hybrid nanocomposites were identified using Fourier Transform Infrared (FTIR) spectrometry. The hybrid composite films were evaluated for mechanical, thermal and morphological characteristics. Morphological results describe a uniform dispersion of silica particles in the polymer matrix. The thermal stability and mechanical properties of polyimide composite were improved, and the decomposition temperature was increased when the amount of silica nanoparticles was increased.
\end{abstract}

Keywords: nanocomposites, nanoparticle modification, hybrid polyimide composites, thermal properties, mechanical properties

\section{Introduction}

Polymer-inorganic hybrid nanocomposites are an emerging class of materials that hold significant promise due to their outstanding properties, which usually arise from a combined effect of the properties of their polymeric and inorganic components [1]. Nanoparticles and nanocomposites are used in a wide range of applications comprising of diverse fields, such as medicine, textiles, cosmetics, agriculture, optics, food packaging, optoelectronic devices, semiconductor devices, aerospace, construction and catalysis [2-4]. Polymeric nanocomposites consisting of inorganic nanoparticles and organic polymers represent a new class of materials that exhibit improved performance compared to their microparticle counterparts [4].

Silica nanoparticles are gaining considerable interest for a wide variety of applications in various fields of material science like electronic, chemical, optical and mechanical industries due to their unique and promising physical and chemical properties [5-7]. The surface modification of nanoparticles in order to change their physical and chemical properties has become an area of significant research in industry. However, the applications of silica nanoparticles are largely limited because of their high energetic surface, which causes the silica nanoparticles to be easily agglomerated. However, this problem could be resolved by using surface modification methods [8]. Surface modification of silica nanoparticles has attracted attention because it produces excellent integration and an improved interface between nanoparticles and polymer matrices [9]. Polymer matrices reinforced with modified silica nanoparticles combine the functionalities of polymer matrices, which include low weight and easy formability,

\footnotetext{
${ }^{*}$ Corresponding author, e-mail: rv.ghorpade@ncl.res.in

(C) BME-PT
} 
with the unique features of the inorganic nanoparticles. However, the nanoparticles have a strong tendency to undergo agglomeration in the polymer matrix, degrading the mechanical properties of the nanocomposites $[4,10,11]$. To improve the dispersion stability of nanoparticles in polymer, it is essential that the particle surface be modified by reactive surface functionalities to generate a strong interaction between monomer precursors and particle surface and repulsion between particles. In order to precisely design desirable surface functionalities, one can select an appropriate surface modification method according to the needs in terms of polymer functional groups and composition.

Also the mechanical properties of these hybrid polymer/nanocomposite materials strongly depend on the dispersion of the reactive sites and on the chemical nature of their environment [12]. This environment mainly consists of the uncovered surface. Therefore, the mode of anchorage of the organic moieties is of prime importance for the characteristics of the final functionalized nanoparticles [13]. Despite the numerous functionalization methods described in literature, only a few allow a precise and easy control of the chemical composition and structure of the surface functionalities of shell. Not all the modification processes offer compatible surface functional groups that can be directly incorporated during polymerization. So, today there is a great challenge to develop alternative methods that provide convenient access to surface functionalized nanoparticles, with the characteristics of the hybrid nanoparticles targeted by the specific property to be achieved.

Aromatic polyimides are important class of thermally stable organic polymers, ranked among the thermally stable polymers. They are widely used in various applications such as high temperature plastics, adhesives, dielectrics, photoresists, nonlinear optical materials and membranes. Additionally, polyimides are used in the fields of aerospace, defense, and optoelectronics; they are also used in liquid crystal alignments, composites, polymer electrolyte fuel cells, fiber optics, etc [14]. The most common technique used to process polyimides is from soluble polyamic acid as a precursor. Films are cast, and then they are thermally dehydrated to produce the final imide form $[14,15]$.

Even though polyimides are thermally very stable polymers, they can still degrade if exposed to a high temperature for a long period. This exposure can lead to changes in the physical and chemical structure of the materials and affect their thermo-oxidative stability [16]. A technology that has been gaining attention in recent years is the incorporation of appropriate nanoscale components into polyimides as a means of improving their thermal and mechanical properties [17-19]. Polyimide-silica materials offer key properties of both components are, therefore, in great demand for high performance applications [20]. There are many factors affecting the properties of hybrid composites, such as the particle size, size distribution, and filler content. In addition, the particle shape and surface functionalities play an important role in the formation of hybrid composite materials [21]. Especially, the bond strength between inorganic particles and polymer matrix should be improved, which always is influenced by the type of dispersion aid and surface functional groups of nanoparticles [22-24]. Thermodynamic immiscibility between inorganic and organic materials, however, may lead to phase separation of the components [25].

Preparation of polymer-inorganic nanocomposites was reported by in situ and ex situ generation of nanoparticles [27, 28]. Sol-gel process [29] is known to generate nanoparticles in situ in the polymerization process, but it is difficult to ensure full control of the shape, crystallinity, size, size distribution, and surface properties of the nanoparticles during the reaction. Also unreacted reactants and byproducts are the major drawback of this process [27]. While, in ex situ process, pre-made and modified nanoparticles are blended with polymer precursors [30], hence, the properties of the nanoparticles can be easily controlled. Therefore ex situ synthetic process of modification and blending of nanoparticles is the simplest and most convenient way to prepare polymer nanocomposites. Polyimide nanocomposites with aliphatic amino functionalized $\mathrm{SiO}_{2}$ nanoparticles were recently reported [28], but the thermal properties like decomposition temperature and $T_{\mathrm{g}}$ deceased with increasing silica content. Therefore the use of suitably surface modified silica nanoparticles is a reasonably good approach for producing fine silica particles uniformly dispersed in the polyimide matrix through polymerization of monomeric precursors with modified silica.

In the present work, simple route was used to modify surface of silica nanoparticle with aromatic amino groups. Polyimide-silica nanocomposites were pre- 
pared using 3,3',4,4'-benzophenone tetracarboxylic dianhydride (BTDA), 4,4'-oxydianiline(ODA) and aromatic amino modified silica nanoparticles by ultrasonic blending followed by thermal imidization. This approach contributes to the novelty in the making of such composite materials. After the surface modification process, the modified silica nanoparticles had better compatibility with polymer matrix and could be well dispersed into the PI matrix in various amounts and the aromatic amino groups on the nanoparticle surface forms covalent bond with the polyimide matrix. Mechanical, thermal and morphological analyses of the composite films were carried out and compared with the film prepared by unmodified silica nanoparticles.

\section{Experimental}

\subsection{Materials}

Chemicals of high purity were obtained from various commercial sources, which consisted of 3,3',4,4'benzophenone tetracarboxylic dianhydride (BTDA; Aldrich), 4,4'-oxydianiline(ODA; Aldrich), Nmethyl-2-pyrrolidone (NMP; Merck), phenyl triethoxysilane (PTEOS; Aldrich). $100 \mathrm{~nm}$ silica nanoparticles were obtained from Fiber Optic Center Inc. USA and dried under vacuum at $120^{\circ} \mathrm{C}$ before use. NMP was purified by distillation under reduced pressure over calcium hydride and stored over $4 \AA$ molecular sieves. Other organic solvents were purified by vacuum distillation. BTDA was dehydrated by drying under a vacuum at $160^{\circ} \mathrm{C}$ for $24 \mathrm{~h}$. The other reagents were used as received.

\subsection{Methods}

The FTIR spectra of silica nanoparticles and silica polymide composite were scanned using a Perkin Elmer 599B spectrophotometer (Waltham, MA) between 400 and $4000 \mathrm{~cm}^{-1}$ using $\mathrm{KBr}$ pellets as medium. XPS measurement was carried out on the APPES system with $\mathrm{MgK}$ radiation $(h r=$ $1253.6 \mathrm{eV}$ ). Differential scanning calorimetry was performed on a TA Q100 DSC instrument to determine the glass transition temperatures $\left(T_{\mathrm{g}}\right)$ of the copolymers. All samples were run against an aluminium reference in crimped aluminium pans. A temperature range of 40 to $400^{\circ} \mathrm{C}$ was used to determine the $T_{\mathrm{g}}$. Two scans were performed on each sample at a heating rate of $10^{\circ} \mathrm{C} / \mathrm{min}$. The second heating results were used for evaluation of $T_{\mathrm{g}}$. Thickness of the film specimens was measured by Mitu- toyo Micrometer. For each individual specimen, measurements were done at five different points and the average value was taken. The thickness data of the film specimens were used for tensile testing. The tensile test was conducted using a Linkam Tensile Stress Testing System-TST 350. Thermogravimetric analysis (TGA) was performed on STA 6000 TGA model from Perkin Elmer instruments. The samples were heated at a rate of $10^{\circ} \mathrm{C} / \mathrm{min}$ under nitrogen atmosphere in the range of 40 to $900^{\circ} \mathrm{C}$. Modified nanosilica and polyimide hybrid composite films were investigated using X-ray diffractometer (Rigaku, Micro Max-007 HF).

\subsection{Modification of silica nanoparticles with PTEOS}

$100 \mathrm{~nm}$ silica nanoparticles were dried under reduced pressure at $120^{\circ} \mathrm{C}$ for $12 \mathrm{~h}$ in vacuum oven. Dried silica nanoparticles $(10.0 \mathrm{~g})$ were suspended in $200 \mathrm{~mL}$ of toluene for $30 \mathrm{~min}$ under sonication. Nanosilicatoluene mixture kept under mechanical stirring in three necked reactor under nitrogen atmosphere. $3.0 \mathrm{~g}$ of triethylamine was added into the mixture. An excess $3.0 \mathrm{~g}$ of phenyl triethoxysilane (PTEOS) was added to a suspension of silica - toluene and stirred for $30 \mathrm{~min}$ at room temperature. The mixture was heated at reflux for $24 \mathrm{~h}$ at high speed stirring. Finally, the particles were washed free of adsorbed initiator using 5 cycles of centrifugation and re-suspension in toluene, and then volatiles were removed under vacuum.

\subsection{Nitration of PTEOS modified nanosilica}

Sodium nitrite $(2.0 \mathrm{~g})$ was added to $100 \mathrm{~mL} \mathrm{RB}$ flask containing a mixture of PTOES modified silica nanoparticles $(2.0 \mathrm{~g})$, chloroform $(20 \mathrm{~mL})$, and acetic acid $(3 \mathrm{~mL})$, and the mixture was stirred for 2 hour at room temperature. Acetic acid $(2 \mathrm{~mL})$ was then added and the mixture was stirred for a further 18 hours. Nanoparticles were separated by centrifugation and washed with water for several times to remove any unreacted $\mathrm{NaNO}_{2}$ and acetic acid. Finally silica nanoparticles were washed by centrifugation with ethanol and dried under vacuum.

\subsection{Reduction of nitro group on modified nanosilica}

$4.0 \mathrm{~g}$ of aromatic-nitro modified silica nanoparticles were dispersed in $50 \mathrm{~mL}$ of ethanol and sonicated for 30 min. $4.0 \mathrm{~g}$ of $\mathrm{SnCl}_{2} \cdot 2 \mathrm{H}_{2} \mathrm{O}$ dissolved in 
$25 \mathrm{~mL}$ of ethanol and added to silica suspension with mechanical stirring at $30^{\circ} \mathrm{C}$. The reaction mixture was kept under stirring for $4 \mathrm{~h}$ at $30^{\circ} \mathrm{C}$. The $\mathrm{pH}$ was made slightly basic $(\mathrm{pH} 8)$ by addition of $5 \%$ aqueous sodium bicarbonate and the resulting basic mixture kept one hour under stirring. Nanoparticles were washed with water for several times to remove unreacted components followed by washing with ethanol and dried under reduced pressure. These modified nanoparticles were characterized by IR, TGA and XPS.

\subsection{Determination of surface amino groups}

A typical non-aqueous titration method was employed to detect the coverage density of amino group on the surface of silica nanoparticles by using $\mathrm{HCl}$ ethanol standard solution. [31] Thymol-blue was used as indicator in the titration, where the referred $\mathrm{HCl}$ was used to quantify the amount of amino groups on the surface of modified nanosilica dispersed in the ethanol. The concentration of surface $-\mathrm{NH}_{2}$ groups were determined which is an average of more than 4 times by this titration process.

\subsection{Polyimide/ modified silica composite}

Polyimide films with various amounts of modified silica nanoparticles were prepared. First, the desired amount of modified silica and NMP were mixed and sonicated for $15 \mathrm{~min}$ (Solution A). Second, the diamine (ODA) was dissolved in NMP and stirred for 15 min (Solution B). Then, the solutions A and $B$ were mixed while stirring was continued for $1 \mathrm{~h}$. Third, the dianhydride (BTDA) was dissolved in Solution $(\mathrm{A}+\mathrm{B})$ with constant stirring. The mixture was stirred for $24 \mathrm{~h}$ at $30^{\circ} \mathrm{C}$. The nanocomposite films were prepared by casting from the viscous poly(amic acid)/modified silica solution on a glass plate using a doctor's knife. The resultant films were dried in an oven and subsequently imidized at a high temperature in air. An imidization program, such as heating from room temperature to $80^{\circ} \mathrm{C}$ in $30 \mathrm{~min}$, heating at $80^{\circ} \mathrm{C}$ for $2 \mathrm{~h}$, heating from 80 to $200^{\circ} \mathrm{C}$ in $5 \mathrm{~h}$, heating from 200 to $300^{\circ} \mathrm{C}$ in $2 \mathrm{~h}$, heating at $300^{\circ} \mathrm{C}$ for $45 \mathrm{~min}$, and then cooling to room temperature, was employed. Table 1 presents the amount of dianhydride, diamine, solvent NMP, modified silica nanoparticles with film thickness of each sample. Polyimide film with $4 \mathrm{wt} \%$ of unmodified silica nanoparticles was prepared as per procedure mentioned.

\section{Results and discussion}

PTEOS was used to modify surface of nanosilica. Aromatic amino functionalized silica nanoparticles were prepared by nitration followed by reduction of PTEOS modified silica nanoparticles as depicted in Figure 1. A typical titration method was employed to detect the surface amino group on the on the modified nanosilica. The density of $-\mathrm{NH}_{2}$ group on the nanoparticle surface is about $180.7 \mu \mathrm{mol} \cdot \mathrm{g}^{-1}$. Also FTIR spectral analysis of nanoparticles confirms modification of silica nanoparticles with aromatic amino groups. Figure 2 shows the FT-IR spectrum of the samples, $\mathrm{SiO}_{2}$ (curve a), PTEOS modified $\mathrm{SiO}_{2}$ nanoparticles (curve b), $\mathrm{SiO}_{2} @ \mathrm{Ar}-\mathrm{NO}_{2}$ (curve c) and $\mathrm{SiO}_{2} @ \mathrm{Ar}-\mathrm{NH}_{2}$ (curve d). In Figure 2 curve a the spectra displayed the characteristic band at $1103 \mathrm{~cm}^{-1}$ of the $\mathrm{Si}-\mathrm{O}-\mathrm{Si}$ unit and symmetric

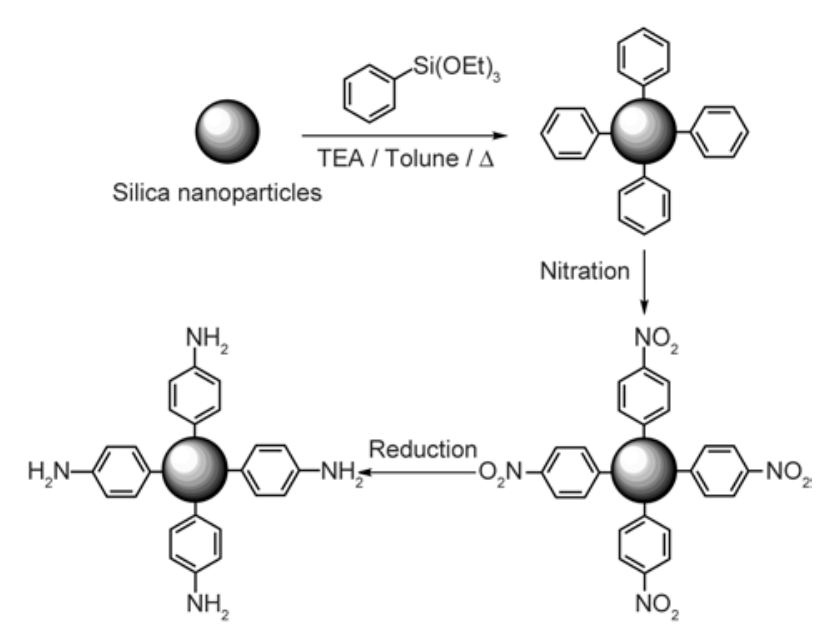

Figure 1. Synthetic scheme for modification of silica nanoparticles

Table 1. Modified silica/ polyimide hybrid film compositions and thickness

\begin{tabular}{|l|c|c|c|c|c|}
\hline Polymer code & $\begin{array}{c}\text { BTDA } \\
{[\mathbf{g}]}\end{array}$ & $\begin{array}{c}\text { ODA } \\
{[\mathbf{g}]}\end{array}$ & $\begin{array}{c}\text { NMP (70 wt\% wrt. of solids) } \\
{[\mathbf{g}]}\end{array}$ & $\begin{array}{c}\text { Modified silica nanoparticles } \\
{[\mathbf{w t} \% \mathbf{g}]}\end{array}$ & $\begin{array}{c}\text { Film thickness } \pm \mathbf{0 . 2} \text { mm } \\
{[\mathbf{m m}]}\end{array}$ \\
\hline PI & 2.0 & 1.24 & 7.56 & $0 / 0$ & 0.162 \\
\hline PIMS-2 & 2.0 & 1.24 & 7.71 & $2.0 / 0.065$ & 0.168 \\
\hline PIMS-4 & 2.0 & 1.24 & 7.86 & $4.0 / 0.130$ & 0.170 \\
\hline PIMS-6 & 2.0 & 1.24 & 8.01 & $6.0 / 0.194$ & 0.165 \\
\hline PIMS-8 & 2.0 & 1.24 & 8.16 & $8.0 / 0.259$ & 0.169 \\
\hline
\end{tabular}




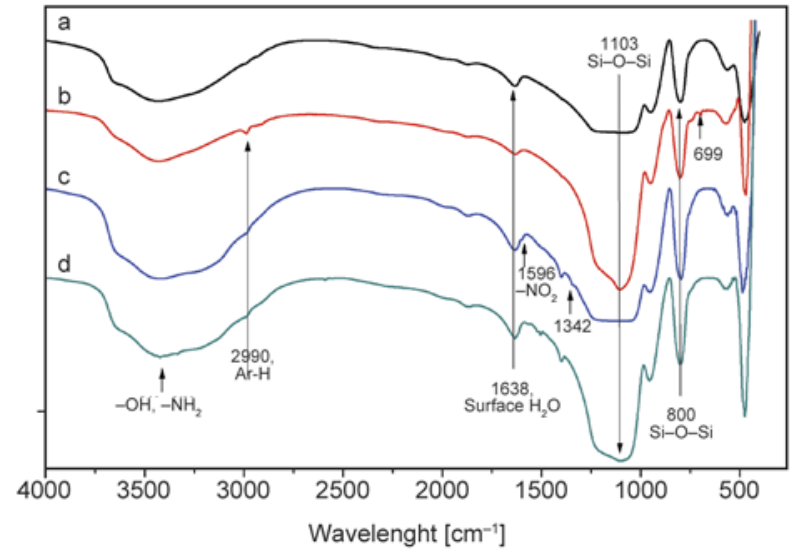

Figure 2. FT-IR spectrum of $\mathrm{SiO}_{2}(\mathrm{a}), \mathrm{SiO}_{2} @ \mathrm{Ar}(\mathrm{b})$, $\mathrm{SiO}_{2} @ \mathrm{Ar}^{-\mathrm{NO}_{2}}$ (c) and $\mathrm{SiO}_{2} @ \mathrm{Ar}-\mathrm{NH}_{2}(\mathrm{~d})$

stretching at $800 \mathrm{~cm}^{-1}$. The broad absorption from 3200 to $3300 \mathrm{~cm}^{-1}$ was assigned to the $\mathrm{Si}-\mathrm{OH}$ linkage. The band at $1638 \mathrm{~cm}^{-1}$ was the bending vibrations of water molecules adsorbed on the surface of the $\mathrm{SiO}_{2}$. Figure 2 curve $\mathrm{b}$ displayed the characteristic features of PTEOS modified $\mathrm{SiO}_{2}$ nanoparticles. Compared with Figure 2 curve a, the weak $\mathrm{C}-\mathrm{H}$ stretching vibration for Ar-H of PTEOS was observed at $2990 \mathrm{~cm}^{-1}$, and the peaks at 699 and $738 \mathrm{~cm}^{-1}$ were due to $\mathrm{Si}-\mathrm{Ph}$ vibrations. In Figure 2 curve $\mathrm{c}$ bands at 1596 and $1342 \mathrm{~cm}^{-1}$ are due to vibrations of nitro groups, indicate nitration of surface aromatic rings. While in Figure 2 curve $d$ disappearance bands at 1596 and $1342 \mathrm{~cm}^{-1}$ and presence of wider band consisted of the asymmetric stretching vibration for $\mathrm{N}-\mathrm{H}$ bonds and the $\mathrm{Si}-\mathrm{OH}$ of $\mathrm{SiO}_{2}$ depicted the reduction of $\mathrm{SiO}_{2} @ \mathrm{Ar}-\mathrm{NO}_{2}$ to $\mathrm{SiO}_{2} @ \mathrm{Ar}-\mathrm{NH}_{2}$.

Modification of silica nanoparticles was also followed by evaluating chemical states of nitrogen using XPS analysis. Figure 3 curve $a$ and curve $b$ shows, the N1 s spectra for $\mathrm{SiO}_{2} @ \mathrm{Ar}-\mathrm{NO}_{2}$ and

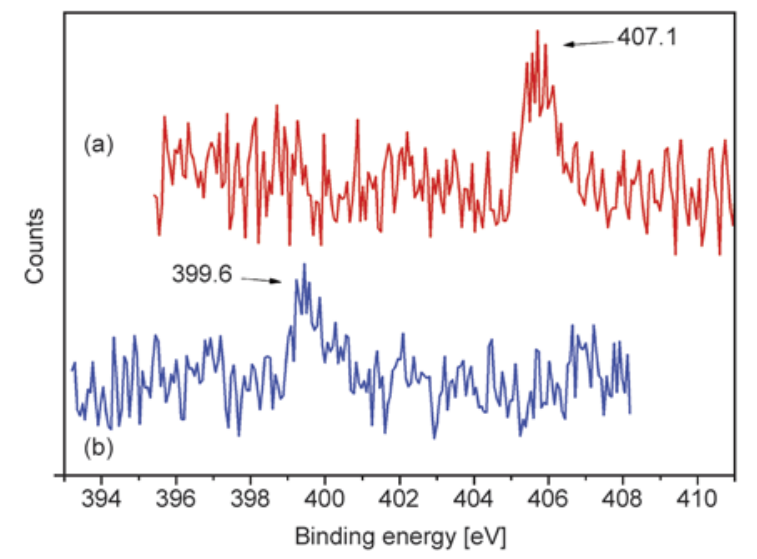

Figure 3. $\mathrm{N} 1 \mathrm{~s}$ XPS spectra of $\mathrm{SiO}_{2} @ \mathrm{Ar}-\mathrm{NO}_{2}$ (a) and $\mathrm{SiO}_{2} @ \mathrm{Ar}^{-\mathrm{NH}_{2}}$ (b)
$\mathrm{SiO}_{2} @ \mathrm{Ar}-\mathrm{NH}_{2}$ nanoparticles respectively. In Figure 3 curve a peak at $407.1 \mathrm{eV}$ and in Figure 3 curve $b$ peak at $399.6 \mathrm{eV}$ attributing to binding energy of $-\mathrm{NO}_{2}$ and $-\mathrm{NH}_{2}$ groups, respectively. Shifting of chemical states of nitrogen confirms that surface $-\mathrm{NO}_{2}$ groups from silica nanoparticles converted into $-\mathrm{NH}_{2}$ groups.

The contact angle measurement was performed to obtain the hydrophilicity/hydrophobicity behavior of nanoparticle surface. The sessile-drop method in air was used to quantify wetting ability, an indicator of hydrophilicity. The contact angle reading taken is the angle between the bordering surfaces, in this case formed between silica monolayer and the water surface. Figure 4 indicates that the water contact angle of aromatic modified silica nanoparticles is higher due to surface hydrophobicity, while the water contact angle decreases on nitration and further decreased on reduction of surface nitro groups to amino groups.

In this study, ODA and BTDA were used to prepare PI matrix. Thin films were obtained from neat polyamic acid as well as its composites with various proportions of modified silica (Figure 5). To prevent aggregation and get uniform dispersion of the nanoparticles, ultrasonic blending was performed on the reaction mixture. The details of the process of silica/PI composite films are shown in Figure 6. The FTIR spectra of neat PI and hybrid composite films are shown in Figure 7. The typical characteristic bands of PI polymer matrix were found. In these spectra, the bands at 1771 and $1710 \mathrm{~cm}^{-1}$ were associated with the imide carbonyl band. In the IR spectra of PIMS-8 additional band at $1104 \mathrm{~cm}^{-1}$ were found due to characteristic vibrations of $\mathrm{Si}-\mathrm{O}$ linkage.

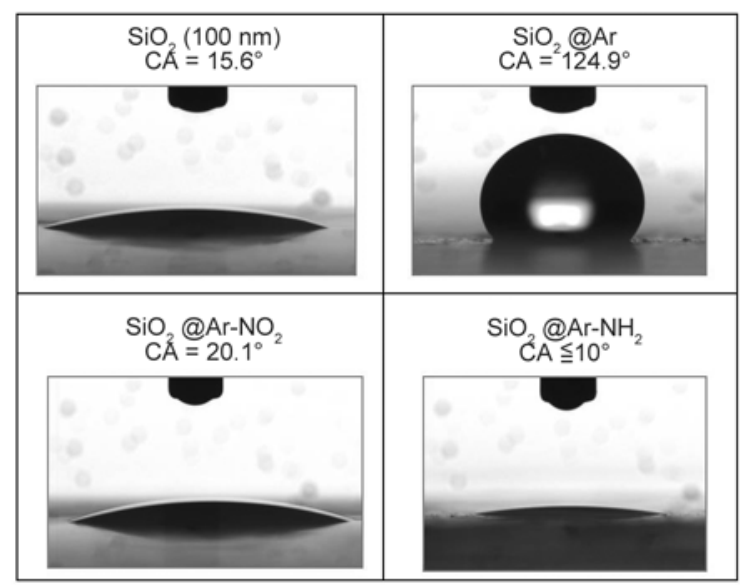

Figure 4. Variation in contact angle during modification of silica nanoparticles 


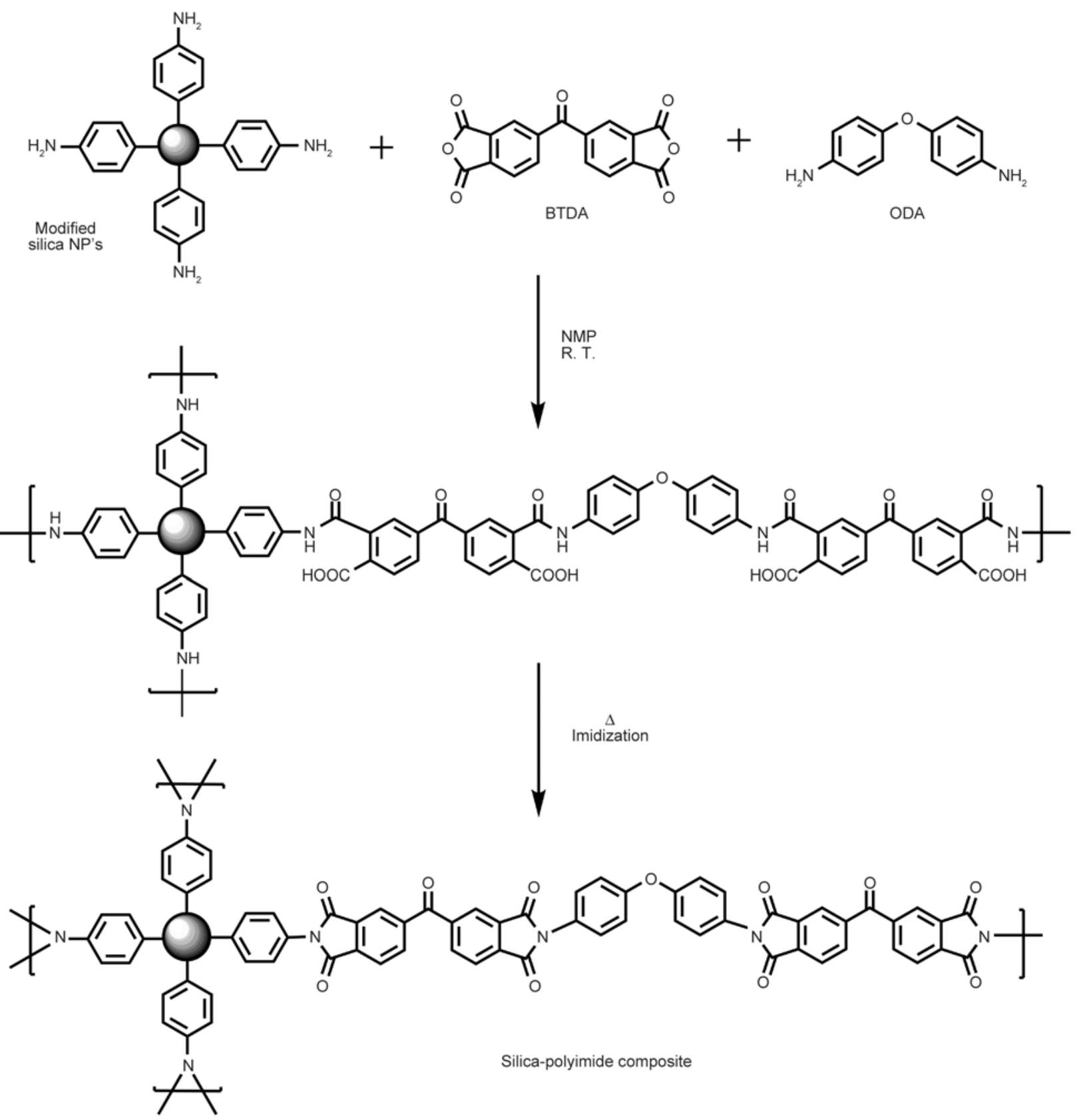

Figure 5. Synthetic scheme of modified silica/polyimide composites

The morphology of the modified silica nanoparticles and hybrid films has been studied using scanning electron microscopy (SEM). After modification with aromatic amino group silica particles remains dispersed and spherical in nature (Figure 8b). The micrographs of the composites containing $4 \mathrm{wt} \%$ modified and unmodified silica in the matrix are shown in Figure 8c and Figure 8d respectively. Composite films have a very homogeneous distribution with no agglomeration of silica in any region across the films. The amino groups present on the surface of nanosilica reacts with polyimide matrix forming covalent bonds and do not allow the agglomeration of silica particles. Such homogeneous distribu- tion of nanoparticles demonstrates better compatibility between modified silica nanoparticles and the polyimide in the composite films. This has resulted in improved strength of the composite films for present system.

To reveal the distribution of silica nanoparticles in the polyimide matrix, transmission electron microscopy (TEM) was performed. The samples for the TEM study were taken from a microtomed section of the polyimide/modified silica nanocomposites. Figure 9 shows TEM micrographs for the polyimide nanocomposites incorporated with $4 \mathrm{wt} \%$ modified silica nanoparticles (Figure 9a) and $4 \mathrm{wt} \%$ unmodified silica nanoparticles (Figure 9b). The light 


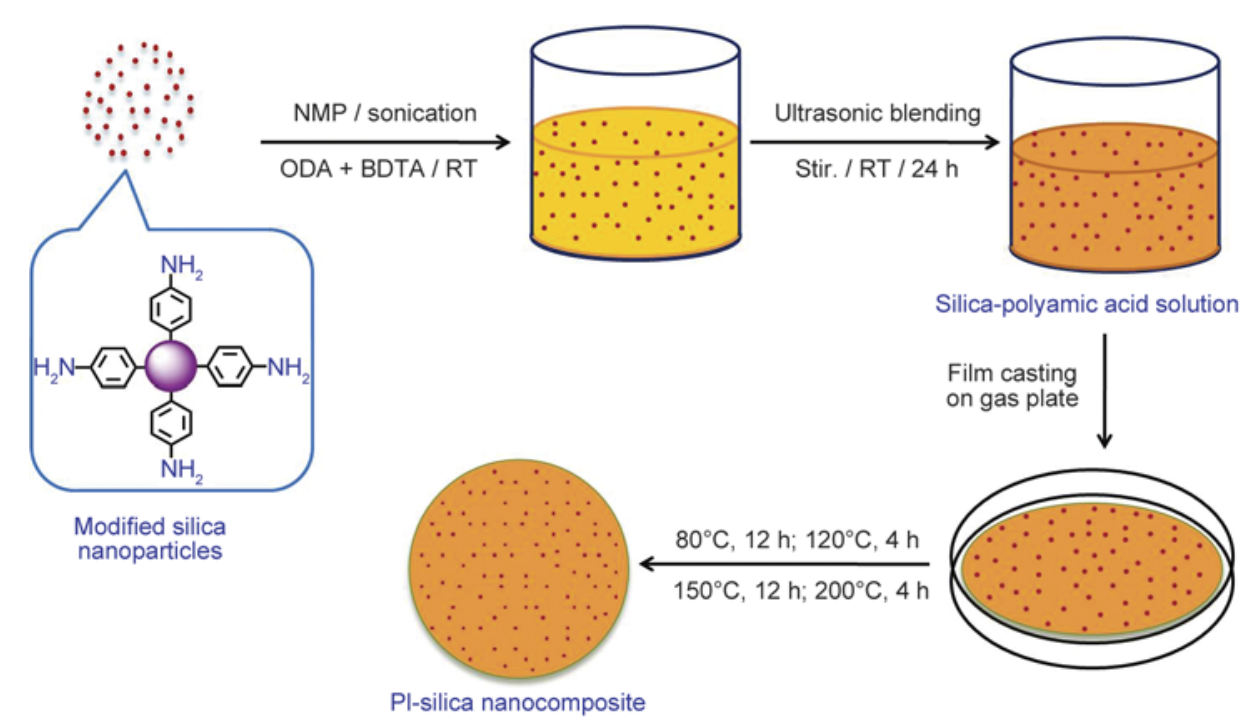

Figure 6. Details of the synthetic process for silica/PI composite

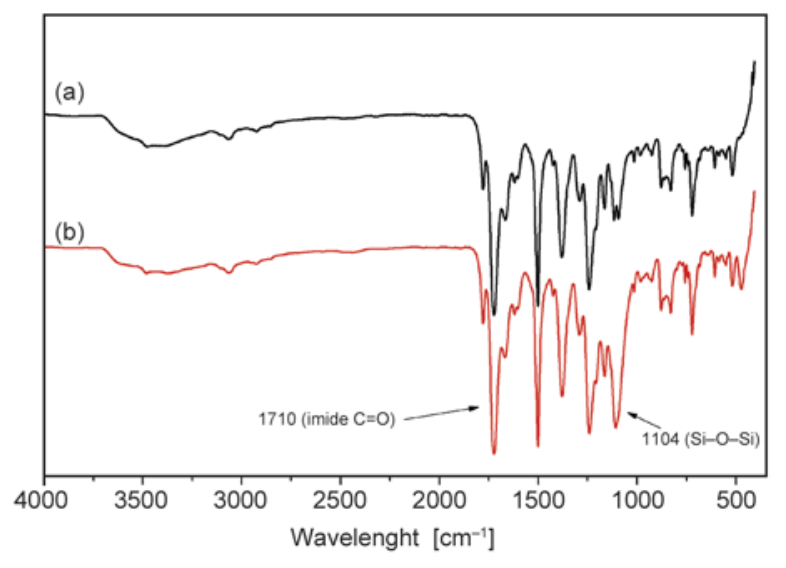

Figure 7. The FT-IR spectra of neat PI (a) and hybrid PI (b) composite films

regions represent the Polyimide matrix, and the gray, dark spots correspond to the $\mathrm{SiO}_{2}$ nanoparticles. The distribution of silica nanoparticles in Figure 9a is more uniform than that in Figure 9b. It can be conclude that aromatic amino modified silica nanoparticles distribute more uniformly in the polyimide matrix than the unmodified silica nanoparticles. It is thought that the modification plays an important role in the uniformity of the silica distribution.

Stress-strain curves of neat polyimide, nanosilica/ polyimide films and modified nanosilica/polyimide films were recorded for evaluation of strain-to-break properties. Dog bone shaped specimens of the neat polyimide and polyimide/modified $\mathrm{SiO}_{2}$ nanocomposites with thickness of around 0.2 micrometer and width of $2.7 \mathrm{~mm}$ at the gauge section were used for the tensile test. The specimen was loaded at a strain rate of $0.1 \mathrm{~mm} / \mathrm{min}$ until the failure happened. The influence of the silica content on the tensile
Table 2. Mechanical properties of modified nanosilica/ polyimide composite films

\begin{tabular}{|l|c|c|}
\hline Polymer code & $\begin{array}{c}\text { Ultimate tensile stress } \\
{[\mathbf{M P a}]}\end{array}$ & $\begin{array}{c}\text { Strain to yield point } \\
{[\mathbf{\%}]}\end{array}$ \\
\hline PI & 82.08 & 8.93 \\
\hline PIMS-2 & 98.72 & 8.80 \\
\hline PIMS-4 & 110.63 & 8.47 \\
\hline PIMS-6 & 122.33 & 7.47 \\
\hline PIMS-8 & 130.42 & 7.00 \\
\hline PIAS-4 & 104.06 & 7.00 \\
\hline
\end{tabular}

strength is shown in Figure 10. The tensile strength at yield point for neat polyimide films was $82 \mathrm{MPa}$. These values of yield stress for the composite films increased (122 MPa) with an increase in the modified silica content up to $8 \mathrm{wt} \%$. The variation of tensile strength at yield point and at break point as a function of modified silica content is illustrated in Table 2. The elongation at break decreased slightly with increasing silica content. Ultimate strength and $\%$ elongation of modified silica/polyimide composite film (PIMS-4) improved compared with unmodified silica/ polyimide composite film (PIAS-4). This may be caused by the fact that the modified nanosilica is much easier to be dispersed homogeneously in the solution and covalently bonded to the polyimide matrix.

The DSC curves presented in Figure 11 shows the evaluation of the glass transition temperatures of nanosilica/polyimide composites. The glass transition temperature of the nanocomposites gradually increased with increase in modified silica content in the polyimide matrix. Because of the surface modification of nanoparticles, the interface between the polymer and the nanoparticle can change and affect 


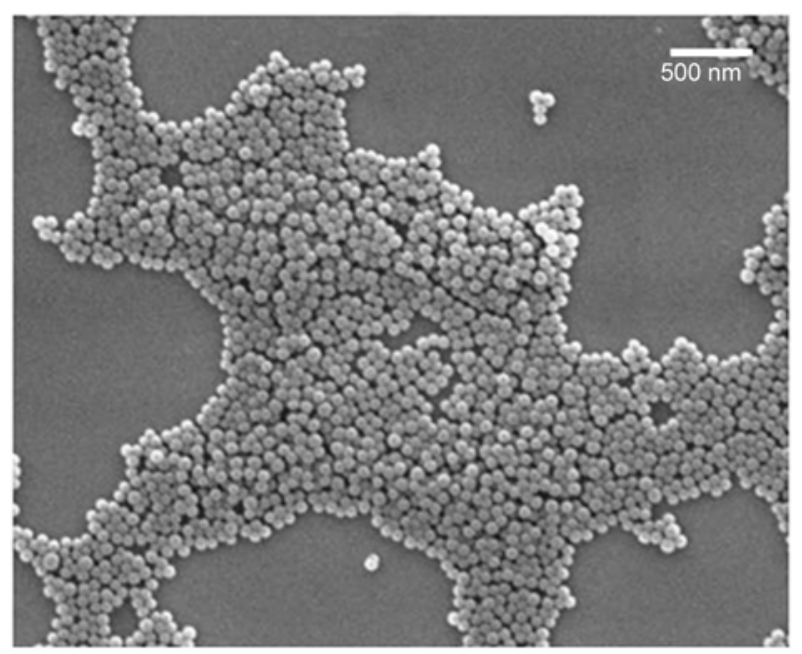

a)

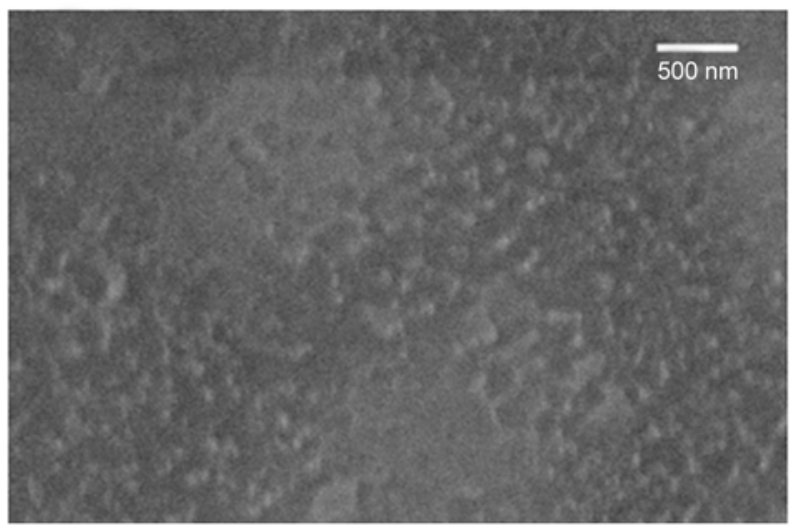

c)

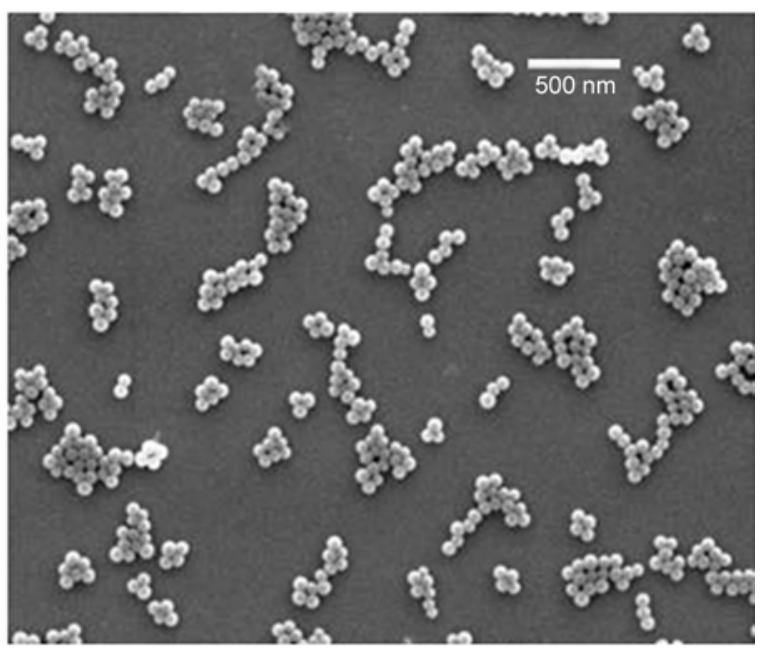

b)

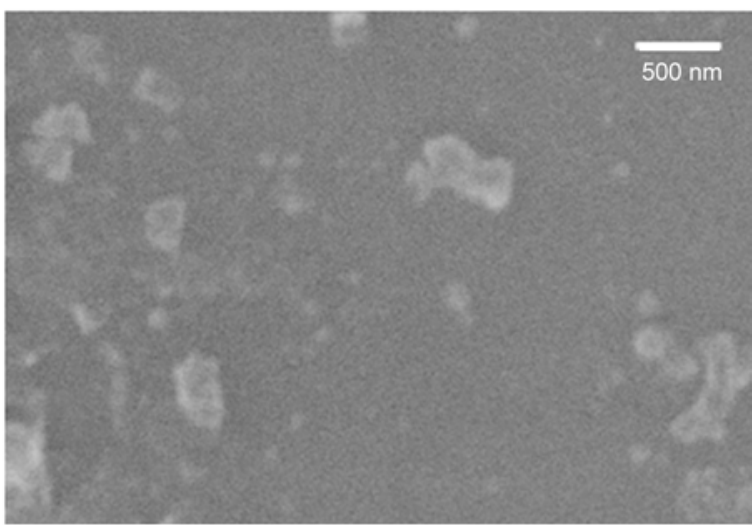

d)

Figure 8. SEM images of silica (a), modified silic (b) modified silica/polyimide (c) and unmodified silica/polyimide (d) composite films

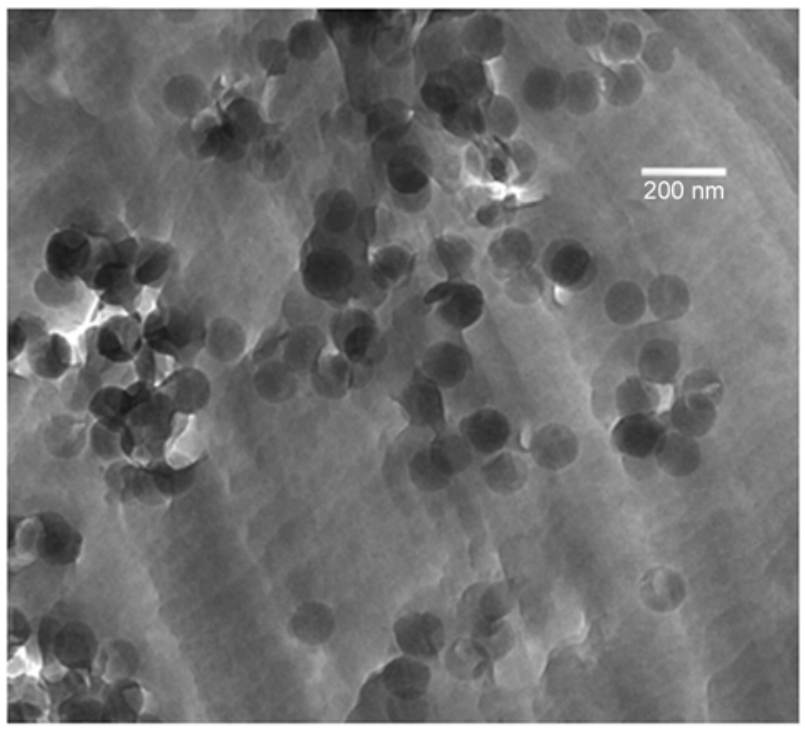

a)

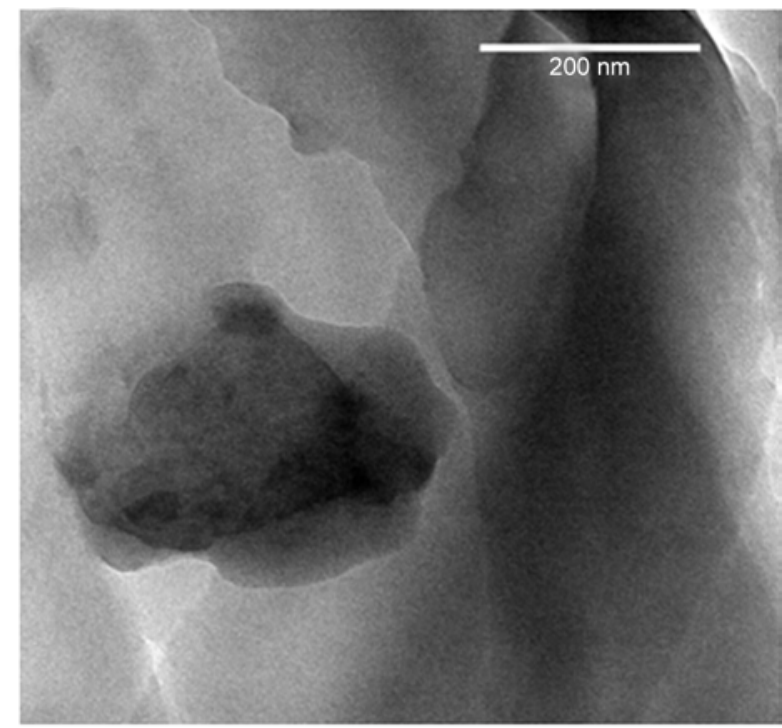

b)

Figure 9. TEM of modified (a) and unmodified (b) silica/polyimide composite films

the thermal properties of the nanocomposites. In this case aromatic amino groups on the nanoparticle surfaces form covalent bonds with polyimide matix and increase the rigidity in the polymer. As a result
$T_{\mathrm{g}}$ increased from 282 to $288^{\circ} \mathrm{C}$ with the $\mathrm{SiO}_{2}$ content increase. Such improvement in $T_{\mathrm{g}}$ is not observed in case of unmodified silica/PI composite. 


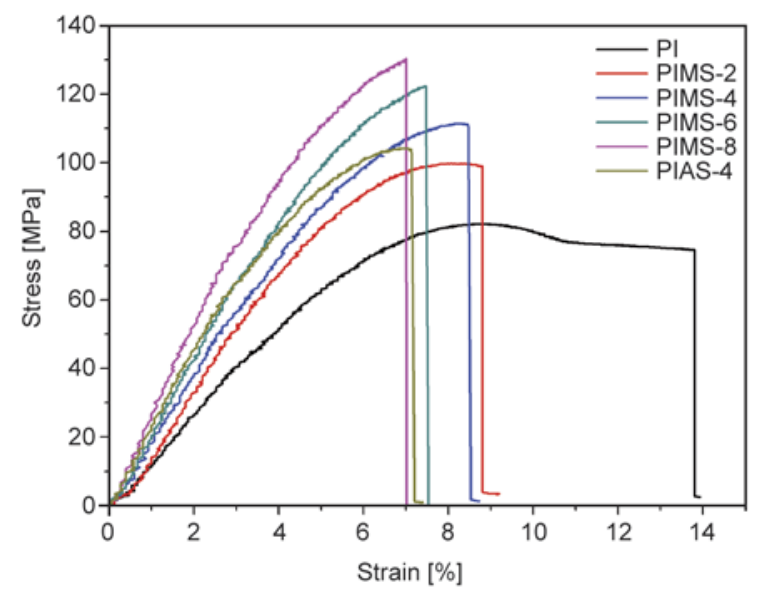

Figure 10. Mechanical tensile testing: stress/strain curves for hybrid polyimide composite films

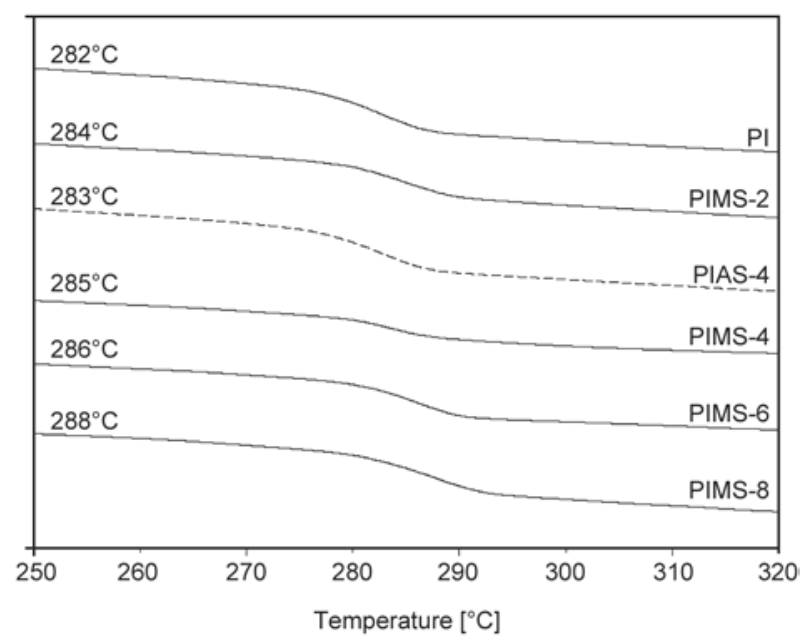

Figure 11. DSC thermograms for silica/ polyimide composite films

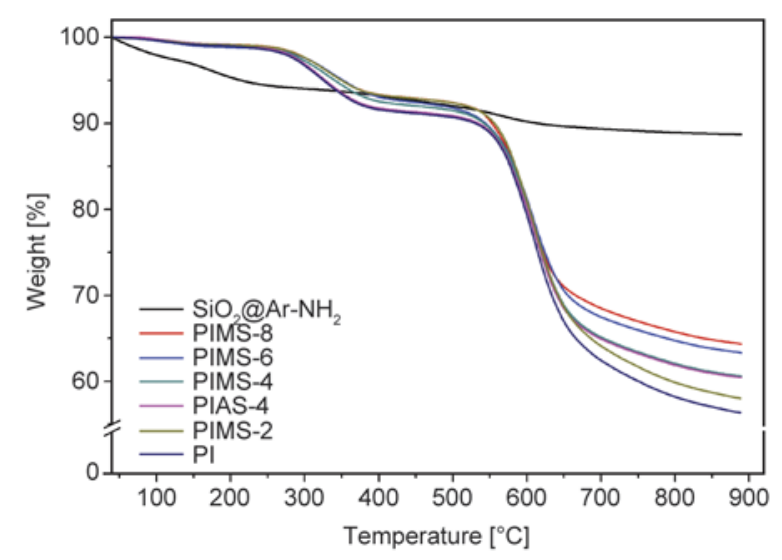

Figure 12. Thermogravimetric analysis of modified silica and hybrid polyimide composite films

The thermal stability of the modified nanosilica/ polyimide composite films was studied by thermogravimetric analysis (TGA). TGA plots of PAI modified nanosilica/polyimide composites with the dif-

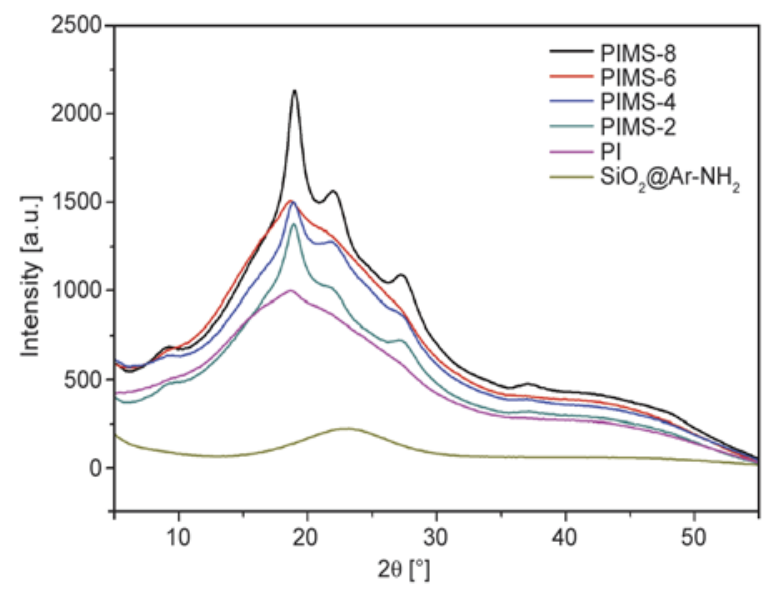

Figure 13. XRD patterns of the modified $\mathrm{SiO}_{2}$ particles and polyimide/ $\mathrm{SiO}_{2}$ composite films

ferent amounts of modified silica nanoparticles are shown in Figure 12. The amount of modified silica nanoparticles to polyimide matrix was changed from 0 to $8 \mathrm{wt} \%$. A weight loss is observed above $150^{\circ} \mathrm{C}$ on all the TGA plots, which corresponds to the loss of water. Weight loss observed at about $250 \sim 350^{\circ} \mathrm{C}$ is due to the trapped solvent as well contribution from imidization arising from surface groups. When the temperature was raised to $500^{\circ} \mathrm{C}$, the polyimide matrix began to decompose and the decomposition temperature became higher when the modified silica nanoparticles were added into the polyimide matrix. At the same temperature, all the curves of the composites indicated that the weight loss of the composites was less than that of the pure polyimide matrix. It is worth pointing out that the thermal stability of polyimide was enhanced with the increasing modified silica contents. Also comparison of thermograms of PIMS-4 and PIAS-4 depicted that, at the initial stage of decomposition, thermal stability of PIMS-4 is higher than PIAS-4, which is again proof for covalent bonding between surface functional amino groups on silica with PI matrix.

The prepared composite films were also characterized by XRD. Figure 13 shows the XRD patterns of the modified $\mathrm{SiO}_{2}$ particles and polyimide $/ \mathrm{SiO}_{2}$ composite films with various contents of modified $\mathrm{SiO}_{2}$. As can be clearly seen in the Figure, peak patterns in the diffractograms of the PI hybrid films reveal semi-crystalline structure polymers. The result indicated that modified nano- $\mathrm{SiO}_{2}$ in polyimide could improve the crystallization behavior of the polymer matrix. 


\section{Conclusions}

The aromatic amino modified silica/PI inorganic/ organic nanocomposite films were prepared by the simple ultrasonic blending, after the silica nanoparticles were modified by aromatic amino groups on surface. In the micrographs of composite films, the modified silica nanoparticles were found to be well dispersed in the PI polymer matrix. The silica nanoparticles were still monodispersed without any agglomerations when the amount of silica nanoparticles to PI reached $8 \mathrm{wt} \%$. The thermal stability of PI improved, and the decomposition temperature was increased with increasing amount of modified nanosilica in hybrid films. The optimum tensile strength was achieved when appropriate amounts of the modified nanosilica used. Tensile measurements indicate that modulus as well as stress at break point improved while elongation at break decreased for the hybrid materials. The shift in $T_{\mathrm{g}}$ values suggests the presence of interactions between the two phases. The morphological investigations reveal a uniform dispersion of silica particles in the polyimide matrix. In this system, the high thermal stability and mechanical strength showed that these modified silica/PI nanocomposite films can be widely used in the high performance polymer applications.

\section{Acknowledgements}

Funding from Department of Science and Technology, Govt. of India (Grant No. SR/S3/CE/0014/2010) is gratefully acknowledged.

\section{References}

[1] Beija M., Marty J-D., Destarac M.: RAFT/MADIX polymers for the preparation of polymer/inorganic nanohybrids. Progress in Polymer Science, 36, 845-886 (2011). DOI: 10.1016/j.progpolymsci.2011.01.002

[2] Trindade T., da Silva D. A.: Nanocomposite particles for bio-applications: Materials and bio-interfaces. Pan Sanford Publishing, Singapore (2011).

[3] Mahfuzn H., Dhanak V.: Nanoparticle reinforced composites for structural applications. Imperial College Press, London (2010).

[4] Kango S., Kalia S., Celli A., Njuguna J., Habibi Y., Kumar R.: Surface modification of inorganic nanoparticles for development of organic-inorganic nanocomposites - A review. Progress in Polymer Science, 38, 1232-1261(2013).

DOI: $10.1016 /$ j.progpolymsci.2013.02.003
[5] O’Farrell N., Houlton A., Horrocks B.: Silicon nanoparticles: Applications in cell biology and medicine. International Journal of Nanomedicine, 1, 451-472 (2006).

DOI: $10.2147 /$ nano.2006.1.4.451

[6] Tang L., Cheng J.: Nonporous silica nanoparticles for nanomedicine application. Nanotoday, 8, 290-312 (2013). DOI: $10.1016 /$ j.nantod.2013.04.007

[7] Taylor R., Coulombe S., Otanicar T., Phelan P., Gunawan A., Lv W., Rosengarten G., Prasher R., Tyagi H.: Small particles, big impacts: A review of the diverse applications of nanofluids. Journal of Applied Physics, 113, 011301/1-011301/19 (2013). DOI: $10.1063 / 1.4754271$

[8] Hu D., Xu Z-L., Chen C.: Polypiperazine-amide nanofiltration membrane containing silica nanoparticles prepared by interfacial polymerization. Desalination, 301, 75-81 (2012).

DOI: $10.1016 /$ j.desal.2012.06.015

[9] Li S., Lin M., Toprak M. S., Kim D. K., Muhammed M.: Nanocomposites of polymer and inorganic nanoparticles for optical and magnetic applications. Nano Reviews, 1, 5214/1-5214/19 (2010).

DOI: $10.3402 /$ nano.v1i0.5214

[10] Yang H., Zhang Q., Guo M., Wang C., Du N., Fu Q.: Study on the phase structures and toughening mechanism in $\mathrm{PP} / \mathrm{EPDM} / \mathrm{SiO}_{2}$ ternary composites. Polymer, 47, 2106-2115 (2006).

DOI: $10.1016 /$ j.polymer.2006.01.076

[11] Kruenate J., Tongpool R., Panyathanmaporn T., Kongrat P.: Optical and mechanical properties of polypropylene modified by metal oxides. Surface and Interface Analysis, 36, 1044-1047 (2004).

DOI: $10.1002 /$ sia. 1833

[12] Hanemann T., Szabó D. V.: Polymer-nanoparticle composites: From synthesis to modern applications. Materials, 3, 3468-3517 (2010). DOI: $10.3390 / \mathrm{ma3} 063468$

[13] Sutra P., Fajula F., Brunel D., Lentz P., Daelen G., Nagy J. B.: ${ }^{29} \mathrm{Si}$ and ${ }^{13} \mathrm{C}$ MAS-NMR characterization of surface modification of micelle-templated silicas during the grafting of organic moieties and end-capping. Colloids and Surfaces A: Physicochemical and Engineering Aspects, 158, 21-27 (1999). DOI: 10.1016/S0927-7757(99)00126-0

[14] Liaw D-J., Wang K-L., Huang Y-C., Lee K-R., Lai JY., Ha C-S.: Advanced polyimide materials: Syntheses, physical properties and applications. Progress in Polymer Science, 37, 907-974 (2012).

DOI: 10.1016/j.progpolymsci.2012.02.005

[15] St. Clair A. K., St. Clair T. L.: Soluble aromatic polyimides for film and coating applications. in 'Polymers for high technology’ (eds.: Bowden M. J., Turne S. R.) American Chemical Society, Washington, 437-444 (1987).

DOI: $\underline{10.1021 / \mathrm{bk}-1987-0346 . c h 037}$ 
[16] Cella J., Ghosh M., Mittal K.: Polyimides: Fundamentals and applications. Marcel Dekker, New York (1996).

[17] Kickelbick G.: Concepts for the incorporation of inorganic building blocks into organic polymers on a nanoscale. Progress in Polymer Science, 28, 83-114 (2003). DOI: 10.1016/S0079-6700(02)00019-9

[18] Bershtein V. A., Egorova L. M., Yakushev P. N., Pissis P., Sysel P., Brozova L.: Molecular dynamics in nanostructured polyimide-silica hybrid materials and their thermal stability. Journal of Polymer Science Part B: Polymer Physics, 40, 1056-1069 (2002).

DOI: $10.1002 /$ polb.10162

[19] Judeinstein P., Sanchez C.: Hybrid organic-inorganic materials: A land of multidisciplinarity. Journal of Materials Chemistry, 6, 511-525 (1996).

DOI: 10.1039/JM9960600511

[20] Khalil M., Saeed S., Ahmad Z.: Mechanical and thermal properties of polyimide/silica hybrids with imidemodified silica network structures. Journal of Applied Polymer Science, 107, 1257-1268 (2008).

DOI: 10.1002/app.27149

[21] Ma X., Lee N-H., Oh H-J., Hwang J-S., Kim S-J.: Preparation and characterization of silica/polyamideimide nanocomposite thin films. Nanoscale Research Letters, 5, 1846-1851 (2010). DOI: $10.1007 / \mathrm{s} 11671-010-9726-7$

[22] Hosseini S., Li Y., Chung T-S., Liu Y.: Enhanced gas separation performance of nanocomposite membranes using $\mathrm{MgO}$ nanoparticles. Journal of Membrane Science, 302, 207-217 (2007).

DOI: $10.1016 /$ j.memsci.2007.06.062

[23] Castellano M., Conzattia L., Costa G., Falqui L., Turturro A., Valenti B., Negroni F.: Surface modification of silica: 1 . Thermodynamic aspects and effect on elastomer reinforcement. Polymer, 46, 695-703 (2005). DOI: $10.1016 /$ j.polymer.2004.11.010
[24] Alexandre M., Dubois P.: Polymer-layered silicate nanocomposites: Preparation, properties and uses of a new class of materials. Materials Science and Engineering R: Reports, 28, 1-63 (2000). DOI: 10.1016/S0927-796X(00)00012-7

[25] Khalil M.: Polyimide/silica hybrids using imide-modified silica network structures. PhD Thesis, Pakistan Institute of Engineering and Applied Sciences, Islamabad (2009).

[26] Lü C., Yang B.: High refractive index organic-inorganic nanocomposites: Design, synthesis and application. Journal of Materials Chemistry, 19, 2884-2901 (2009).

DOI: $10.1039 / \mathrm{b} 816254 \mathrm{a}$

[28] Kim Y-J., Kim J-H., Ha S-W., Kwon D., Lee J-K.: Polyimide nanocomposites with functionalized $\mathrm{SiO}_{2}$ nanoparticles: Enhanced processability, thermal and mechanical properties. RSC Advances, 4, 4337143377 (2014).

DOI: $10.1039 / \mathrm{c} 4 \mathrm{ra} 04952 \mathrm{~g}$

[29] Schottner G.: Hybrid sol-gel-derived polymers: Applications of multifunctional materials. Chemistry of Materials, 13, 3422-3435 (2001).

DOI: $10.1021 / \mathrm{cm} 011060 \mathrm{~m}$

[30] Barbey R., Lavanant L., Paripovic D., Schüwer N., Sugnaux C., Tugulu S., Klok H-A.: Polymer brushes via surface-initiated controlled radical polymerization: Synthesis, characterization, properties, and applications. Chemical Reviews, 109, 5437-5527 (2009). DOI: $10.1021 / \mathrm{cr} 900045 \mathrm{a}$

[31] Hu Y., Zhang Y., Tang Y.: One-step hydrothermal synthesis of surface organosilanized nanozeolite under microwave irradiation. Chemical Communications, 46, 3875-3877 (2010). DOI: $10.1039 / \mathrm{C} 002551 \mathrm{H}$ 